\title{
Random vibration based damage detection for a composite beam under environmental and operational variability via a stochastic Functional Model based method
}

\author{
Tryfon-Chrysovalantis Aravanis ${ }^{1}$, Spiros Kolovos ${ }^{1}$, John Sakellariou ${ }^{1, *}$, and Spilios Fassois ${ }^{1}$ \\ ${ }^{1}$ Stochastic Mechanical Systems \& Automation (SMSA) Laboratory \\ Department of Mechanical Engineering \& Aeronautics \\ University of Patras 26504, Greece
}

\begin{abstract}
The problem of random vibration response based damage detection for a composite beam under non-measurable environmental and operational variability, presently temperature and tightening torque, is considered via a Functional Model based method. The method is based on proper representation of the healthy structural dynamics under any environmental/operating conditions via a data based Functional Model obtained in the method's baseline phase and used to define a 'healthy subspace'. Damage detection is, in the method's inspection phase, achieved by examining whether or not the current dynamics belongs to the healthy subspace. The experimental results obtained for damage detection on a composite beam indicate excellent detection performance, with correct detection rate of $100 \%$ for false alarm rate as small as $1 \%$. The superiority of the proposed method is confirmed via comparisons with a state-of-the-art Principal Component Analysis based method.
\end{abstract}

\section{Introduction}

For many in-service structures the variability in their dynamics due to varying environmental and/or operating conditions, such as temperature, wind, boundary conditions and so on, may be so significant as to 'mask' changes due to damage. Vibration based Structural Health Monitoring (SHM), which aims at detecting damage via changes it induces on the dynamics [1], may then be subject to poor performance (lack of robustness to the varying conditions), characterized by low detection rate and/or high false alarm rate.

The problem of vibration based SHM robustness may be accounted for by attempting separation of the effects of damage from those of varying environmental/operating conditions on the dynamics. In cases where the varying environmental/operating conditions may be continuously measured during the normal operation of the structure, their effects in the dynamics may be counteracted via cause-and-effect type modeling, allowing for their separation from those of damage [2]. Yet, the continuous, in operation measurement of the actual conditions is often not feasible. Alternative damage detection methods [3], usually employing Principal Component Analysis (PCA) and related techniques, are based on the selection of characteristic quantities representing the dynamics (for instance natural frequencies), their transformation, and subsequent decomposition into two groups: one affected by the varying environmental/operating conditions and the other by potential damage, so that damage detection may be then achieved based on the latter group. Other methods postulate the complete

\footnotetext{
*e-mail: sakj@mech.upatras.gr
} 
(typically probabilistic) modeling of the healthy structural dynamics under all potential environmental/operating conditions, so as to then better distinguish the effects of damage [4-6].

The goal of the present study is the low and limited bandwidth random vibration based

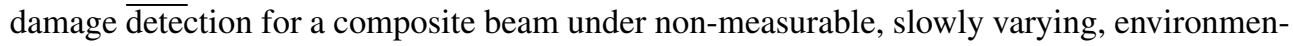
tal and operating conditions via a recently introduced Functional Model (FM) robust detection method [7]. The method is based on representing, in a baseline (learning) phase, the healthy structural dynamics under all environmental and operating condition of interest in a proper subspace, referred to as the 'healthy subspace'. In the inspection phase, damage detection is achieved by examining whether or not the current (unknown health state) dynamics belongs to this 'healthy subspace'. The 'healthy subspace' is constructed via a data based Vector-dependent Functionally Pooled AutoRegressive with eXogenous excitation (VFP-ARX) model that incorporates the variable conditions via a proper operating (scheduling) parameter vector.

The method's effectiveness for damage detection on a composite beam under slowly varying environmental and operating conditions is then considered. The beam employed represents the topology of a commercial Unmanned Aerial Vehicle (UAV) boom, with the varying environmental and operating conditions being temperature and tightening torque on the fuselage side, respectively. Two damage scenarios are simulated via the addition of small masses on the surface of the beam, while special adhesive tape is also used for simulating material and/or manufacturing uncertainty, such as variation in the resin, fiber orientation and so on. The attained damage detection performance is also compared to that of a state-of-the-art Principal Component Analysis based method [4].

The rest of this paper is organized as follows: The precise problem statement is presented in Section 2. The Functional Model based method for robust damage detection is briefly presented in Section 3. The structure, the varying environmental/operating conditions, the damage scenarios, and the experiments are presented in Section 4, while the method's performance assessment and comparison in Section 5. Concluding remarks are finally summarized in Section 6.

\section{Precise problem statement}

The damage detection problem tackled may be stated as follows: Consider a structure operating under slowly varying environmental/operational conditions, which are represented in quantitative form by a vector $\boldsymbol{k}$, of dimensionality equal to the number of independent variability sources.

In the baseline (learning) phase of the method (implemented once at an initial stage with the structure in its healthy state), $M$ low and limited bandwidth random vibration response signal pairs $\left(x_{\boldsymbol{k}}[t], y_{\boldsymbol{k}}[t]\right)(t$ designating discrete, normalized by the sampling period, time, $t=$ $1,2, \ldots, N)$ are obtained from $M$ distinct samples of the environmental/operational conditions collected in the vectors, $\boldsymbol{k}_{1}, \boldsymbol{k}_{2}, \ldots \boldsymbol{k}_{M}{ }^{1}$, that is:

Baseline phase: $\left(x_{\boldsymbol{k}}[t], y_{\boldsymbol{k}}[t]\right)$ for $t=1,2, \ldots, N$ and under various environmental/operating conditions $\boldsymbol{k}=\boldsymbol{k}_{1}, \boldsymbol{k}_{2}, \ldots \boldsymbol{k}_{M}$ for the healthy structural state.

Then, in the inspection (diagnosis) phase, during the method's normal operation, given a single fresh pair of random vibration signals, $\left(x_{u}[t], y_{u}[t]\right)^{2}$, that is:

Inspection phase: $\left(x_{u}[t], y_{u}[t]\right)$ for $t=1,2, \ldots, N$ under unknown environmental/operating conditions (unknown $\boldsymbol{k}$ ) and with the structure being in unknown health state,

determine whether or not the structure is in its healthy state.

Note that the environmental/operating conditions may be generally different in the inspection phase from those used in the baseline phase, although it is desirable that the former

\footnotetext{
${ }^{1}$ Vector/matrix quantities are designated by bold face lower/upper characters, respectively.

${ }^{2}$ The subscript ' $u$ ' designating unknown structural health state.
} 
belong to the range defined by the latter. Moreover, note that all vibration response signals are of the same nature (displacement, velocity or acceleration), measured at specific locations with a uniform sampling period $T_{s}$, with each signal pair being measured under constant environmental/operating conditions ${ }^{3}$. Finally, the method, in its current form, applies to structures characterized by linear and time invariant dynamics.

\section{The stochastic Functional Model based method for robust damage detection}

Baseline (learning) phase ('healthy subspace' construction). The 'healthy subspace' is represented via a stochastic Functional Model (FM) that models the healthy structural dynamics under all considered environmental and operating conditions [7]. The specific FM employed in this study is a Vector-dependent Functionally Pooled AutoRegressive with eXogenous excitation (VFP-ARX) model with proper operating (scheduling) parameter vector (details below).

For its construction, a total number of $M$ controlled experiments - under measured environmental/operating conditions - are performed for a sample of the environmental/operating conditions of interest. Each experiment is conducted under a specific value of the quantity representing the conditions considered (presently temperature and tightening torque on the mounting), with the complete series of experiments covering the range $\left[k_{\min }^{c}, k_{\max }^{c}\right] \times$ $\left[k_{\min }^{\tau}, k_{\text {max }}^{\tau}\right]$ via the discretization:

$$
k^{c} \in\left\{k_{1}^{c}, k_{2}^{c}, \ldots, k_{M_{1}}^{c}\right\}, \quad k^{\tau} \in\left\{k_{1}^{\tau}, k_{2}^{\tau}, \ldots, k_{M_{2}}^{\tau}\right\}
$$

with the superscripts $c, \tau$ designating temperature and torque, respectively, and the subscript discretization index. Thus, for an experiment under a specific combination of temperature and torque, the operating parameter vector $\boldsymbol{k}=\left[k_{l}^{c} k_{m}^{\tau}\right]^{T}\left(l=1,2, \ldots M_{1}, m=1,2, \ldots M_{2}\right)$ is employed.

The complete set of baseline experiments then provides $M=M_{1} \times M_{2}$ random vibration response signal pairs $\left(x_{\boldsymbol{k}}[t], y_{\boldsymbol{k}}[t]\right)^{4}$, each signal being $N$ samples long $(t=1,2, \ldots, N)$. Based on this set, a VFP-ARX $\left(n_{a}, n_{b}\right)_{p}$ of the form [8]:

$$
\begin{aligned}
y_{\boldsymbol{k}}[t]+\sum_{i=1}^{n_{a}} a_{i}(\boldsymbol{k}) \cdot y_{\boldsymbol{k}}[t-i] & =\sum_{i=0}^{n_{b}} b_{i}(\boldsymbol{k}) \cdot x_{\boldsymbol{k}}[t-i]+e_{\boldsymbol{k}}[t], \quad e_{\boldsymbol{k}}[t] \sim i i d \mathcal{N}\left(0, \sigma_{e}^{2}(\boldsymbol{k})\right), \quad \boldsymbol{k} \in \mathbb{R}^{2} \\
a_{i}(\boldsymbol{k}) & =\sum_{j=1}^{p} a_{i, j} \cdot G_{j}(\boldsymbol{k}), \quad b_{i}(\boldsymbol{k})=\sum_{j=1}^{p} b_{i, j} \cdot G_{j}(\boldsymbol{k})
\end{aligned}
$$

is obtained with $n_{a}, n_{b}$ designating the $\mathrm{AR}$ and $\mathrm{X}$ orders, respectively, iid identically independently distributed, $\mathcal{N}$ Gaussian distribution and $e_{\boldsymbol{k}}[t]$ is the innovations (model residual) signal under conditions $\boldsymbol{k}$, assumed to be zero-mean, white (serially uncorrelated) with variance $\sigma_{e}^{2}(\boldsymbol{k})$ and potentially cross-correlated with their counterparts corresponding to different environmental/operating conditions (different $\boldsymbol{k}$ 's). As indicated by Eqs. (2b), the AR and X parameters are modeled as explicit functions of $\boldsymbol{k}$, by using a $p$-dimensional functional subspace spanned by the (mutually independent) functions $G_{1}(\boldsymbol{k}), G_{2}(\boldsymbol{k}), \ldots G_{p}(\boldsymbol{k})$. These form a functional subspace basis that consists of bivariate polynomials obtained as tensor products from typical univariate polynomials such as Legendre, Chebyshev and so on. The constants $a_{i, j}$ and $b_{i, j}$ designate the AR and $\mathrm{X}$ coefficients of projection, respectively.

\footnotetext{
${ }^{3}$ This implies that changes in the environmental/operating conditions occur slowly, at intervals much longer than the acquired signal duration.

${ }^{4}$ The structural dynamics are then employed through the transmittance function in a response-only sense [6].
} 
The determination of the VFP-ARX model orders and its functional subspace dimensionality, for a given basis function family, is based on standard procedures using a Genetic Algorithm (GA) for the minimization of the Bayesian Information Criterion (BIC), while model validation is based on formal verification of the residual sequence uncorrelatedness (whiteness) hypothesis corresponding to the $M$ experiments used in model estimation; VFP-ARX model estimation and validation details are provided in [8].

Inspection (diagnosis) phase. Damage detection is achieved by examining, via a fresh random vibration signal pair $\left(x_{u}[t], y_{u}[t]\right)(t=1,2, \ldots, N)$, obtained under unknown environmental and operating conditions (that is unknown current value of $\boldsymbol{k}$ ), whether or not the structural dynamics reside within the 'healthy subspace', so that the structure may or may not, respectively, be declared as healthy. This is essentially equivalent to examining whether or not the current signal pair $\left(x_{u}[t], y_{u}[t]\right)$ is 'consistent' with the available VFP-ARX model expressing the 'healthy subspace'. This 'consistency' examination may be realized in two steps:

Step 1: Employ the VFP-ARX model to estimate the unknown environmental/operating condition vector $\boldsymbol{k}$ that 'best' (according to a proper criterion) expresses the current signal pair. That is, given the current random vibration signal pair $\left(x_{u}[t], y_{u}[t]\right)$, the estimates ${ }^{5} \hat{\boldsymbol{k}}$ and $\widehat{\sigma}_{e}^{2}(\widehat{\boldsymbol{k}})$ (innovations variance) are obtained using the VFP-ARX model of Eqs. (2a), (2b), as follows ${ }^{6}$ :

$$
\widehat{\boldsymbol{k}}=\arg \min _{\boldsymbol{k}} \sum_{t=1}^{N} e_{u}^{2}[t, \boldsymbol{k}], \quad \widehat{\sigma}_{e}^{2}(\widehat{\boldsymbol{k}})=\frac{1}{N} \sum_{t=1}^{N} e_{u}^{2}[t, \widehat{\boldsymbol{k}}]
$$

Step 2: Using the obtained value of $\boldsymbol{k}, \widehat{\boldsymbol{k}}$, 'consistency' is confirmed following successful validation of the model corresponding to $\widehat{\boldsymbol{k}}$. This is based on a standard statistical test for determining residual $\left(e_{u}[t, \widehat{\boldsymbol{k}}]\right)$ whiteness at a user selected risk level via the Peña-Rodríguez test $D$ statistic which follows a standard normal distribution for a white sequence [9]. If this model is properly validated, the structure is declared as healthy, otherwise as damaged. It is noted that in the case of a healthy declaration, correct estimates of the current environmental/operating conditions are provided by $\widehat{\boldsymbol{k}}$.

\section{The structure, the varying environmental/operating conditions, the damage scenarios and the experiments}

The structure. As already mentioned, the structure is a composite beam representing the topology of one (of two) boom of a twin-tail commercial Unmanned Aerial Vehicle (UAV). In the experimental set-up it is clamped at one end, simulating its connection to the fuselage, while its free end is attached to an aluminum mass representing the aircraft tail (Figures 1(a),(b)). The beam has square hollow cross section, dimensions $600 \times 65 \times 65 \mathrm{~mm}$ (LX $\mathrm{W} \times \mathrm{H}$ ), wall thickness of $3 \mathrm{~mm}$, corner radius of $8 \mathrm{~mm}$ (Figure 1(c)) and mass of $633 \mathrm{~g}$. The beam has been manufactured based on one shot Resin Transfer Moulding (RTM) using 11 plies of twill ( $3 \mathrm{~K}$ carbon fiber fabric of $200 \mathrm{~g} / \mathrm{m}^{2}$ ) and unidirectional (12K carbon fiber fabric of $300 \mathrm{~g} / \mathrm{m}^{2}$ ) weave carbon fiber patterns.

The varying environmental/operating conditions and the damage scenarios. The beam is placed in a freezer, with temperature varying in the range $[0-28]^{\circ} \mathrm{C}$, while the tightening torque on Bolt A (Figure 1(b)) varies from 1 up to $4 \mathrm{Nm}$, simulating assembly uncertainty. Two 'damage' scenarios are simulated: Damage $D_{1}$ corresponds to an added mass of $4.5 \mathrm{~g}$, and $D_{2}$ to an added mass of $12.6 \mathrm{~g}$; in all cases the mass is placed at Point D on the beam (Figure 1(b)). A small additional uncertainty is introduced via a small or larger piece of special (reinforced by plastic mesh) adhesive tape placed (in some experiments) at Point $\mathrm{T}$ on

\footnotetext{
${ }^{5}$ Estimators/estimates are designated by a hat.

${ }^{6} e_{u}[t, k]$ corresponds to the residual $e_{\boldsymbol{k}}[t]$ in Eq. (2a).
} 

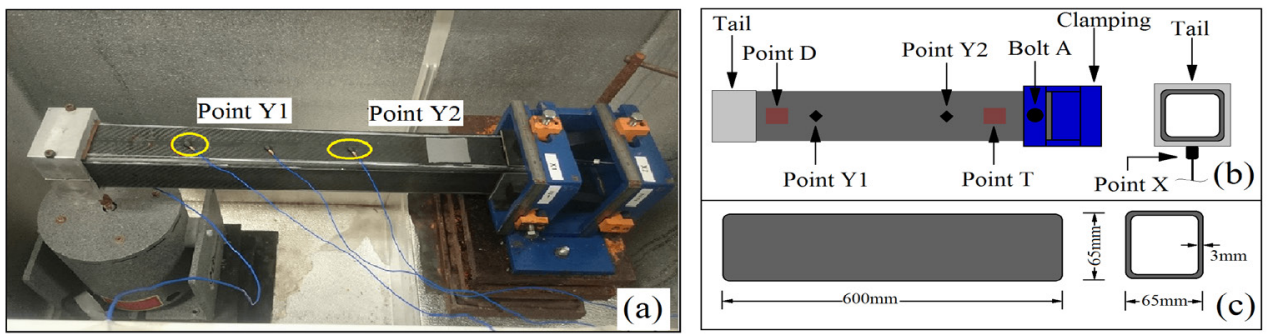

Figure 1. The beam and the experimental set-up. (a) Photo of the set-up. (b) Schematic of the beam with the damage position (Point $\mathrm{D}$ ), adhesive tape position (Point T), excitation position (Point $\mathrm{X}$ ), and vibration measurement positions (Points Y1 and Y2) indicated. (c) Geometric details.

Table 1. Experimental details.

\begin{tabular}{|c|c|c|c|}
\hline Structural state & $\begin{array}{l}\text { Temperature } \\
\text { range }\left({ }^{\circ} C\right)\end{array}$ & $\begin{array}{c}\text { Torque } \\
(\mathrm{Nm})\end{array}$ & $\begin{array}{l}\text { No. of } \\
\text { exps }\end{array}$ \\
\hline \multicolumn{4}{|c|}{ Baseline (learning) phase } \\
\hline Healthy & Set $\mathrm{A}:=\left[0^{\circ}-28^{\circ}\right]$ with a step of $2^{\circ}$ & $1,2,3,4$ & $60^{*}$ \\
\hline \multicolumn{4}{|c|}{ Inspection (diagnosis) phase } \\
\hline \multirow[t]{4}{*}{ Healthy $(H)$} & $\mathrm{A} \&\left\{3^{\circ}, 21^{\circ}\right\}$ & 1 & $51^{\diamond}$ \\
\hline & $\mathrm{A} \&\left\{9^{\circ}, 19^{\circ}, 25^{\circ}\right\}$ & 2 & $54^{\diamond}$ \\
\hline & $\mathrm{A} \&\left\{15^{\circ}, 25^{\circ}\right\}$ & 3 & $51^{\diamond}$ \\
\hline & $\mathrm{A} \&\left\{3^{\circ}, 9^{\circ}, 15^{\circ}, 19^{\circ}, 21^{\circ}\right\}$ & 4 & $60^{\diamond}$ \\
\hline Healthy $\left(H_{1}\right)$ (small tape) & 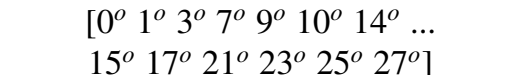 & $1,3,4$ & $117^{\diamond}$ \\
\hline Healthy $\left(H_{2}\right)$ (large tape) & $-/ /-$ & $-/ /-$ & $-/ /-$ \\
\hline Damaged $\left(D_{1}\right)(4.5 \mathrm{~g}$ mass $)$ & $-/ /-$ & $-/ /-$ & $-/ /-$ \\
\hline Damaged $\left(D_{2}\right)(12.6 \mathrm{~g}$ mass $)$ & $-1 /-$ & $-/ /-$ & $-/ /-$ \\
\hline \multicolumn{4}{|c|}{$* 1$ experiment per temperature and torque value. } \\
\hline \multicolumn{4}{|c|}{$\checkmark 3$ experiments per temperature and torque value. } \\
\hline \multicolumn{4}{|c|}{ Sampling frequency $f_{s}=4654.5 \mathrm{~Hz}$; signal length 2500 samples $(0.54 \mathrm{~s}) ; \mathrm{BWD}[5-2327.25] \mathrm{Hz}$} \\
\hline
\end{tabular}

the surface of the beam (Figure 1(b)) and simulating potential material and/or manufacturing variability (such as variation in resin, fiber orientation, and so on).

The experiments. Vibration experiments are performed using an electromechanical shaker applying a random, low frequency band limited white, Gaussian force vertically at Point $\mathrm{X}$, while the acceleration response signals at Points Y1 and Y2 on the beam are acquired through lightweight $(0.5 \mathrm{~g})$ accelerometers (Figures 1(a),(b)). Experiments are performed with the healthy beam under 60 distinct environmental and operating conditions (temperature and torque sets) for the training of the method (baseline phase; details in Table 1). Likewise, 684 experiments are conducted with the structure under healthy or damaged condition for the method's assessment in the inspection phase (details in Table 1). Each measured vibration response signal is sample mean corrected and normalized by its own sample standard deviation.

Preliminary analysis. Welch-based estimates [10, pp. 186-187] of the transmittance function (TF) magnitude with the healthy beam under various environmental and operating conditions, reveal significant variability in the dynamics (Figures 2(a),(b)). Additional TF magnitude estimates for the healthy and damaged beam, under various conditions, are presented in Figure 2(c), from which it is obvious that the variability present in the healthy dynamics 'masks' the effects of the smaller damage $D_{1}$ almost everywhere within the $0-1200 \mathrm{~Hz}$ fre- 

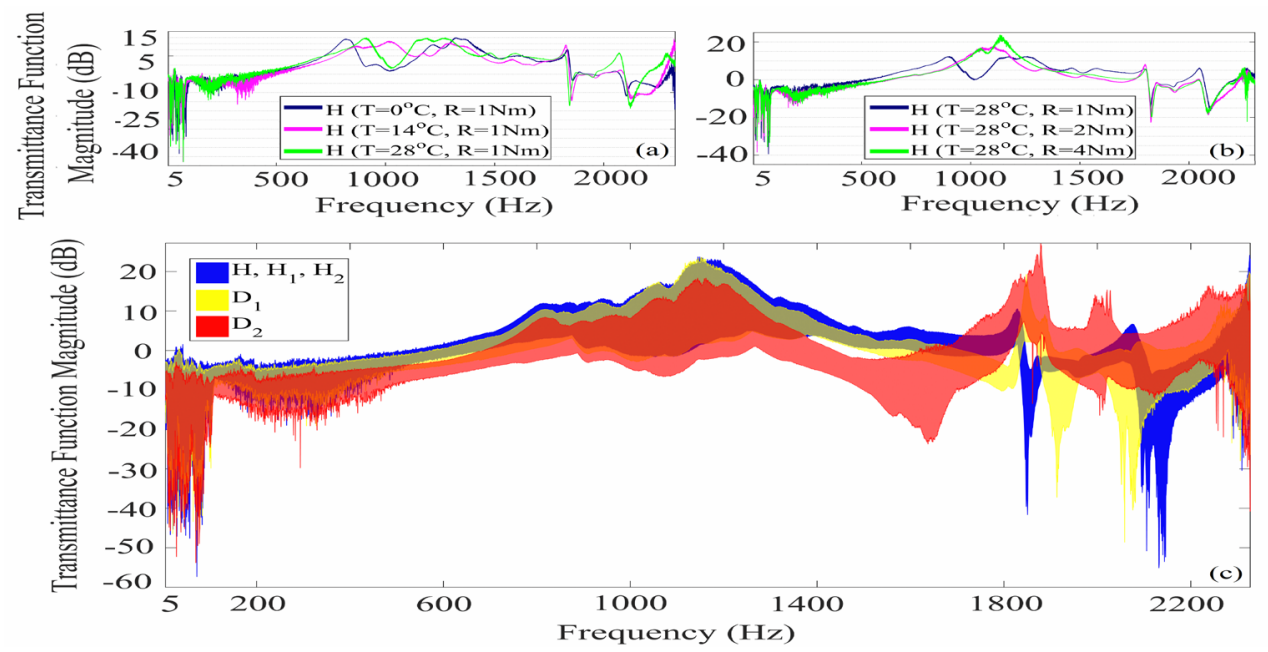

Figure 2. Assessment of the healthy transmittance dynamics under various conditions (a), (b), and the healthy and damaged dynamics under various conditions $\left(H, H_{1}, H_{2}: 450\right.$ experiments, $D_{1}: 117$ experiments, $D_{2}: 117$ experiments) (c). (Welch based estimates based on $N=100000$ sample-long signals, Hamming windowing, 8192 sample-long segments, 95\% overlap.)

quency range, thus pinpointing a challenging damage detection problem. On the other hand, more obvious deviations between the healthy and damaged dynamics are exhibited for the larger damage $D_{2}$.

\section{Experimental results}

\subsection{Damage detection results}

Baseline (learning) phase. Vibration response signals from Points Y1 and Y2 obtained from a single experiment with the healthy beam under specific values of temperature $\left(12{ }^{\circ} \mathrm{C}\right)$ and tightening torque $(1 \mathrm{Nm})$ are used for the estimation of a conventional ARX model based on standard identification procedures [10, pp. 203-205]. This leads to an $\operatorname{ARX}(70,70)$ model characterized by zero delay ( $b_{0} \neq 0$ in the exogenous polynomial). Maintaining the AR and $\mathrm{X}$ orders along with zero delay within the set of VFP-ARX models, the functional subspace is determined using $M=60$ experiments (see Table 1) and a GA based optimization procedure [8]. This leads to a VFP-ARX $(70,70)_{30}$ model with functional subspace consisting of $p=30$ bivariate Shifted Legendre polynomials (estimation details in Table 2).

Inspection (diagnosis) phase. Detection performance is assessed based on 117 experiments per damage scenario under various temperatures and tightening torque values, as well as 450 experiments with the healthy beam; details in Table 1 . All conditions are distinct from those used in the baseline phase.

The results, in terms of the $D$ statistic involved in the Peña-Rodríguez test and ROC curves $^{7}$ are, for both damage scenarios, provided in Figures 3(a1),(a2). Evidently, the healthy test cases are always separated from the smaller damage $D_{1}$, and almost always from the larger one $D_{2}$. The excellent results are reflected in the ROC curves, indicating a detection rate of $100 \%$ even for $0 \%$ false positive (false alarm) rate for damage $D_{1}$, and even for $1 \%$ (or greater) false positive (false alarm) rate for damage $D_{2}$.

\footnotetext{
${ }^{7}$ A Receiver Operating Characteristic, ROC, curve [11, pp. 34-35] represents the true positive rate, that is percentage of correct damage detections, versus the false positive (false alarm) rate for varying detection threshold.
} 
Table 2. Functional Model based method details.

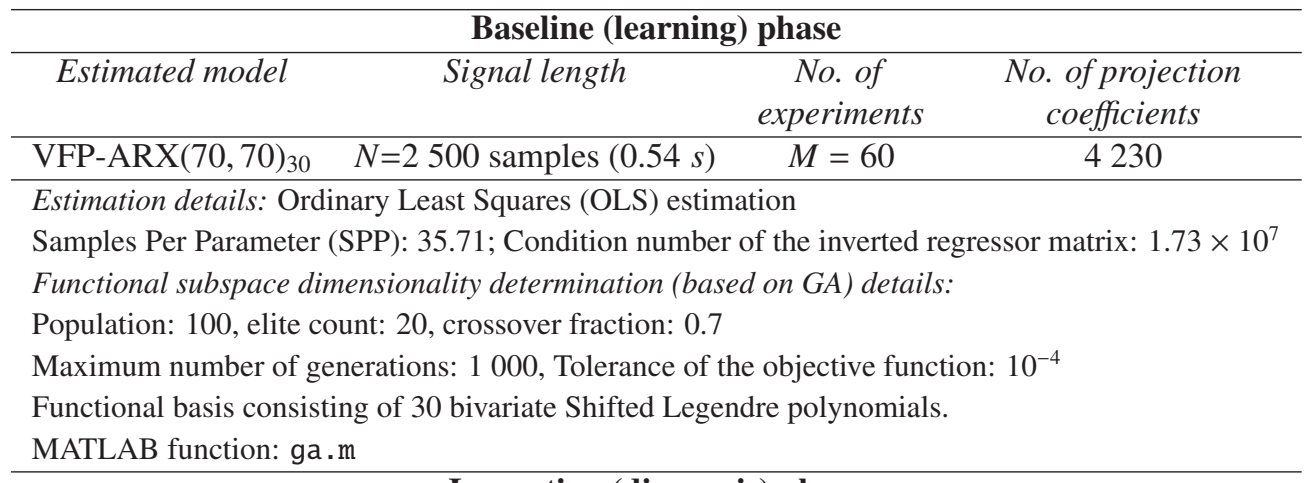

\section{Inspection (diagnosis) phase}

Estimation details: NLS estimator based on golden search and parabolic interpolation

Tolerance of the objective function: $10^{-10}$; Tolerance of the estimated value: $10^{-10}$

MATLAB function: fminbnd.m
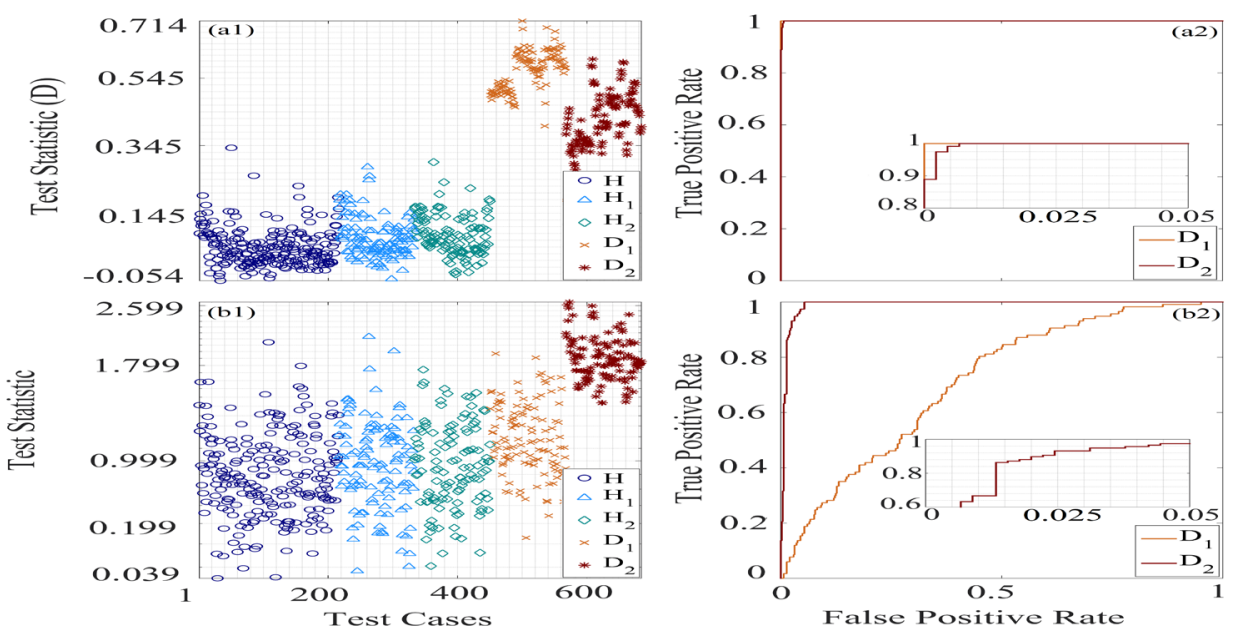

Figure 3. Damage detection assessment results: (a) The Functional Model based method (left: test statistic D, right: ROC curves). (b) The U-PCA-ARX method (left: test statistic, right: ROC curves). (Each plot includes results from 450 experiments with the healthy beam and 117 per damage scenario.)

\subsection{Comparison with the U-PCA-ARX method}

An interesting comparison with the Unsupervised PCA based method using ARX modeling (U-PCA-ARX) [4] is presently made.

Baseline (learning) phase. The pairs of vibration response signals from the same 60 experiments used in the FM method's baseline phase are employed for the estimation of 60 transmittance function $\operatorname{ARX}(70,70)$ models [6], say $m_{o, i}(i=1, \ldots, 60)$, with corresponding parameter vectors $\boldsymbol{a}_{o}=\left\{\boldsymbol{a}_{o, 1}, \ldots, \boldsymbol{a}_{o, 60}\right\}$. The estimated parameter vector sample mean and covariance matrix are then obtained, with each, centered around zero by subtracting their sample mean. The covariance matrix is then decomposed as $\boldsymbol{P}=\boldsymbol{U} \boldsymbol{S}^{2} \boldsymbol{U}^{T}$, with $\boldsymbol{S}^{2}$ designating a diagonal matrix containing the (positive) eigenvalues (set in decreasing order) and $\boldsymbol{U}$ the matrix containing the respective eigenvectors. PCA involves keeping only the last $n$ eigenvectors of the eigenvector matrix $\boldsymbol{U}$, which are presumably associated with damage, 
while dropping the first $q$ which explain a certain fraction $\gamma(\%)$ of the total parameter vector variability and are presumably associated with environmental and operating conditions. The resulting eigenvector sub-matrix is then used for the transformation of the centered parameter vectors $\boldsymbol{a}_{o}=\left\{\boldsymbol{a}_{o, 1}, \ldots, \boldsymbol{a}_{o, 60}\right\}$ into the reduced $n$-dimensionality space [4]; these being also referred to as the 'healthy set'.

The employed sample covariance matrix is of dimensionality $50 \times 50$, corresponding to a vector consisting of the first $25 \mathrm{AR}$ and $25 \mathrm{X}$ parameters. As the number of variability sources affecting the structural dynamics is presently known to be two (temperature and tightening torque), a reasonable number of components to be removed is $q=2$, leading to $n=48$ and $\gamma=45.5 \%$.

Inspection (diagnosis) phase. Once a fresh pair of vibration response signals is obtained from the current (in unknown health state) structure, a fresh transmittance function ARX model $m_{u}$ (with parameter vector $\boldsymbol{a}_{u}$ ) is estimated, and its parameters are centered by subtracting the sample mean obtained in the baseline phase. $\boldsymbol{a}_{u}$ is subsequently transformed into the reduced $n$-dimensionality space, and damage detection is based on a pseudo-statistic involving its Euclidean distance from the 'healthy set'; details are provided in [4].

Damage detection results via the U-PCA-ARX method are presented in Figures 3(b1),(b2) in terms of the test pseudo-statistic and ROC curves. The method reaches a correct detection rate of $\sim 97 \%$ for damage $D_{2}$ for a false positive rate of $\sim 5 \%$ (or greater), while its performance for the smaller damage $D_{1}$ is inadequate. In both cases, it is clear that the method's performance is inferior to that of the Functional Model based method. It has been further observed that the performance may improve by increasing $\gamma$ to $99.6 \%$, yet it remains inferior to that of the Functional Model based method.

\section{Conclusions}

The problem of low bandwidth random vibration based damage detection for a composite beam under non-measurable environmental and operational variability has been investigated via a Functional Model based method. The method's performance, assessed using hundreds of test cases, has been found to be essentially ideal, and significantly better than that of a state-of-the-art PCA based method.

This research was supported by Grant (56990000) from the Research Committee of the University of Patras via 'K. Karatheodori' program

\section{References}

[1] S.D. Fassois, J.S. Sakellariou, J. Royal Society, Series A, 365, 1851, 411-448 (2007)

[2] J. D. Hios, S. D. Fassois, Smart Mater. and Struct. 18, 035011, 1-15 (2009)

[3] A. M. Yan, G. Kerschen, P. De Boe, J. C. Golinval, Mech. Syst. Signal Proc., 19, 847-864 (2005)

[4] K. Vamvoudakis-Stefanou, J.S. Sakellariou, S.D. Fassois, Mech. Syst. Signal Proc., 111, 149-171 (2018)

[5] K. Vamvoudakis-Stefanou, J.S. Sakellariou, S.D. Fassois, Proc. Intern. Conf. on Noise and Vibr. Engin. (ISMA2016), Leuven, Belgium (2016)

[6] A.G. Poulimenos, J.S. Sakellariou, J. Struct. Health Monit. (2018, in press)

[7] T.-C.I. Aravanis, J.S. Sakellariou, S.D. Fassois, Proc. Surveillance 9, Fes, Morocco (2017)

[8] C.S. Sakaris, J.S. Sakellariou, S.D. Fassois, Mech. Syst. Signal Proc. 74, 199-213 (2016)

[9] D. Peña, J. Rodríguez, J. Statist. Planning and Inference, 136, 8, 2706-2718 (2006)

[10] L. Ljung, System Identification: Theory for the User, (Prentice-Hall, New Jersey, 1999)

[11] R. Duda, P. Hart, D. Stork. Pattern Classification, (Wiley, New Jersey, 2000) 